\title{
Musique et possession dans les candomblés de Bahia : pluralisme rituel et comportemental
}

\section{Xavier Vatin}

\section{(2) OpenEdition}

\section{Journals}

Édition électronique

URL : http://journals.openedition.org/ethnomusicologie/116

ISSN : 2235-7688

Éditeur

ADEM - Ateliers d'ethnomusicologie

Édition imprimée

Date de publication : 1 novembre 2006

Pagination : 191-209

ISSN : 1662-372X

Référence électronique

Xavier Vatin, « Musique et possession dans les candomblés de Bahia : pluralisme rituel et comportemental », Cahiers d'ethnomusicologie [En ligne], 19 | 2006, mis en ligne le 15 janvier 2012, consulté le 01 mai 2019. URL : http://journals.openedition.org/ethnomusicologie/116

Ce document a été généré automatiquement le 1 mai 2019

Tous droits réservés 


\title{
Musique et possession dans les candomblés de Bahia : pluralisme rituel et comportemental
}

\author{
Xavier Vatin
}

$1 \quad$ Les candomblés de Bahia ont fait l'objet de nombreuses études dans diverses disciplines des sciences sociales. Toutefois, le phénomène de la possession demeure un point de vive controverse. Les théories formulées, longtemps marquées d'un profond ethnocentrisme ou de l'allégeance à différents courants anthropologiques, ont donné des explications univoques et partielles quant à son origine, sa nature et son induction, explications dont la somme met en évidence la complexité d'un phénomène que seule une perspective transdisciplinaire saura, peut-être, décrypter. Nous allons tenter ici de voir comment s'élaborent, dans les candomblés de Bahia, les relations de la musique et de la possession, selon le contexte rituel et le type d'entité censé s'incarner.

2 La musique tient en effet une place centrale dans la plupart des cultes de possession, si bien que cette dernière a souvent été tenue pour résultat direct de l'action physiologique, voire neurophysiologique des tambours, dont on dénote la présence dans les contextes rituels les plus divers. Toutefois, les recherches menées dans le domaine de l'ethnomusicologie tendent à montrer que le lien entre musique et possession est sans doute de nature plus symbolique que physiologique ${ }^{1}$. La possession s'inscrit dans un carcan rituel et comportemental extrêmement codifié. C'est en tant que code culturellement défini - et non par le biais d'un mystérieux pouvoir intrinsèque - que la musique permet d'induire la possession; c'est en ce qu'elle représente et non en ellemême qu'elle possède donc ce pouvoir. Comme l'affirme Roger Bastide: "La transe africaine ou afro-américaine est un langage (à la fois moteur et vocal) qui se décrypte selon un certain code; il a son vocabulaire, ses règles grammaticales et sa syntaxe » (Bastide 1972 : 96).

3 Dans le candomblé, la musique - composée de centaines de chants, accompagnés de rythmes joués par trois tambours et une cloche métallique - structure le déroulement des 
cérémonies rituelles. Chaque divinité possède un répertoire spécifique de chants; certaines divinités ont aussi des formules rythmiques personnalisées et toutes se caractérisent par des pas de danse particuliers, qui diffèrent plus ou moins d'une "nation ${ }^{2}$ " de candomblé à l'autre. Aux yeux des adeptes, la musique contribue à l'établissement de liens entre le monde des humains et celui des divinités, en d'autres termes au déclenchement puis à la conduite de la possession. La musique se situe par conséquent au cœur d'un système qui met en action les représentations symboliques, spirituelles et religieuses de toute la communauté.

Selon l'ethnographie traditionnelle, dans le candomblé, ce ne sont pas les hommes qui se rendent chez les habitants du monde invisible - comme ce serait le cas du chamanisme mais au contraire les divinités qui descendent sur terre en prenant possession des initiés rituellement préparés à les recevoir. Toutefois, nous allons voir que cette vision dichotomique du chamanisme et de la possession peut, dans certains cas, conduire à une impasse typologique, lorsqu'on tente, par exemple, d'y introduire une opposition binaire entre " musiquant » et « musiqué », pour reprendre la terminologie de Gilbert Rouget.

La possession, dans son contexte rituel - nous verrons que ce n'est pas forcément le cas dans un contexte domestique -, est intimement liée à la musique et à la danse, dont elle est souvent tenue pour résultat direct. Selon Rouget, «Un rituel de possession est une architecture du temps qui comporte [...] différentes phases auxquelles s'attachent différentes musiques » (Rouget 1990 : 89). Il affirme également que le possédé n'est pas le «musiquant» de sa propre transe et ajoute:« La logique [...] du système veut que, foncièrement, le possédé ne soit ni musicien, ni musiquant, mais musiqué » (ibid.: 215). Nous reviendrons sur cette affirmation, en tentant de rendre compte de la diversité des comportements des possédés, d'une "nation» de candomblé à l'autre, d'un type d' « entité » à l'autre (orixá, vodum, inquice, erê, caboclo, exu ; voir glossaire). En effet, selon le contexte rituel, le comportement de l'adepte avant, pendant et après la possession est extrêmement polymorphe. Même si chaque initié manifeste un certain degré d'idiosyncrasie, il est toutefois possible de mettre en évidence des stéréotypes comportementaux qui caractérisent chaque culte et chaque type d'« entité » susceptible de s'incarner. Ces modalités comportementales sont généralement regroupées, si l'on se réfère aux catégories employées par Rouget, sous la dénomination de «transe de possession », au cours de laquelle le possédé n'est que le «musiqué » de sa transe - par opposition au chamane « musiquant » - et ne s'exprime que par la danse.

Or, il s'avère qu'un inventaire détaillé de ces comportements met en évidence des relations très diverses entre musique et possession, remettant partiellement en question une perspective globalisante d'inspiration structuraliste. La même personne, selon l'esprit qu'elle incarne, peut se comporter, d'une cérémonie à l'autre, voire au cours d'une même cérémonie, soit en "musiquant» de sa propre transe, soit exclusivement en " musiqué ». Même s'il est particulièrement difficile de rendre compte de cette diversité, une description minutieuse semble en mesure de l'appréhender. Tentons de suivre le déroulement de la possession dans son contexte cérémoniel, en distinguant différentes étapes : la survenue de la possession, en énumérant quels en sont les "déclencheurs " potentiels; les manifestations qui accompagnent l'« entrée en transe " (nous verrons à cet égard que la terminologie vernaculaire est bien plus riche que celle dont nous disposons pour qualifier ces différentes manifestations) ; le comportement du possédé ; le retour à l'« état normal ». 


\section{Les « déclencheurs » de la possession}

7 Pour que la possession d'un initié survienne, il faut que de nombreuses conditions contextuelles soient réunies; lorsqu'elles le sont, il est toutefois indéniable que certains éléments, de natures diverses - sonores, visuels, olfactifs - peuvent être qualifiés de «déclencheurs» de la possession. Pour les officiants, il s'agit donc d'«appeler " la divinité (chamar o santo), en employant un ou plusieurs de ces déclencheurs.

\section{Déclencheurs sonores}

\section{Un ou plusieurs chants spécifiques}

Des chants spécifiques peuvent être utilisés pour déclencher la possession chez un, plusieurs, voire tous les initiés à la fois. Ces chants sont généralement appelés cantigas de fundamento (chant de «fondement») ou cantiga de chamar o santo (« chant pour appeler le saint »). On en trouve dans les trois principales nations, mais leur usage et leurs effets varient sensiblement d'une nation à l'autre. Dans la nation Ketu, ces chants forment un vaste répertoire et la plupart d'entre eux sont associés à une divinité spécifique; de ce fait, lorsqu'on en chante un, ce sont surtout les initiés dont la divinité principale (santo de cabeça) est alors " appelée » qui sont susceptibles de « répondre », c'est-à-dire d'entrer en transe. Les possessions se succèdent donc selon l'ordre des divinités invoquées. Dans la nation Angola, un seul chant - choisi par le chanteur soliste parmi un répertoire plus restreint de chants, que les adeptes nomment chamadas (appels) - suffit à déclencher la possession chez tous les initiés susceptibles d'être possédés à une occasion déterminée ${ }^{3}$. Les possessions surviennent donc toutes ensemble, en général à la fin de la première partie de la cérémonie, dont la fonction est de susciter la venue des divinités. Dans la nation Jêje, les possessions surviennent en général avant la cérémonie - le matin qui la précède, au cours d'un rite réservé à quelques membres de la communauté - de sorte que les initiés arrivent déjà possédés dans le barracão. Luiza da Rocha, célèbre mère de saint de la nation Jêje Mahi récemment disparue, affirmait à cet égard qu'un seul mot lui suffisait à déclencher la possession chez les initiés. Toutefois, dans cette même nation, certains chants ont aussi pour effet d'induire la possession. Dans le cas des cérémonies pour les caboclos, esprits d'indiens divinisés, il existe des chants "d'appel»; ceux-ci peuvent être entonnés par le chanteur soliste, mais aussi par un caboclo - pour provoquer la venue d'un autre caboclo - puisque, comme nous allons le voir, les adeptes possédés par des caboclos chantent, et sont ainsi les « musiquants » de leur propre transe.

\section{Une ou plusieurs formules rythmiques spécifiques}

Ces formules rythmiques, jouées seules - c'est-à-dire sans chant - sont appelées toques de fundamento, littéralement "rythmes de fondement ». La nation Ketu possède plusieurs toques de fundamento, chacun associé à une divinité particulière. La nation Jêje en possède un nommé adarrum, que l'on retrouve, sous forme d'emprunt, dans les autres nations. Rarement exécuté, il a pour effet de déclencher très efficacement la possession chez tous les initiés, quelle que soit leur divinité et indépendamment des conditions évoquées plus haut quant à la survenue de la possession. La nation Angola possède une formule rythmique nommée barravento, servant généralement d'accompagnement aux chants 
mais qui, lorsqu'elle est jouée seule dans des circonstances précises, a alors pour fonction de déclencher la possession chez de nombreux initiés.

Fig. 1 : Initiée agitant la cloche sacrée adjá; à gauche, initié incarnant la divinité Oxossi. Salvador (Bahia), 1994. Photo Xavier Vatin.

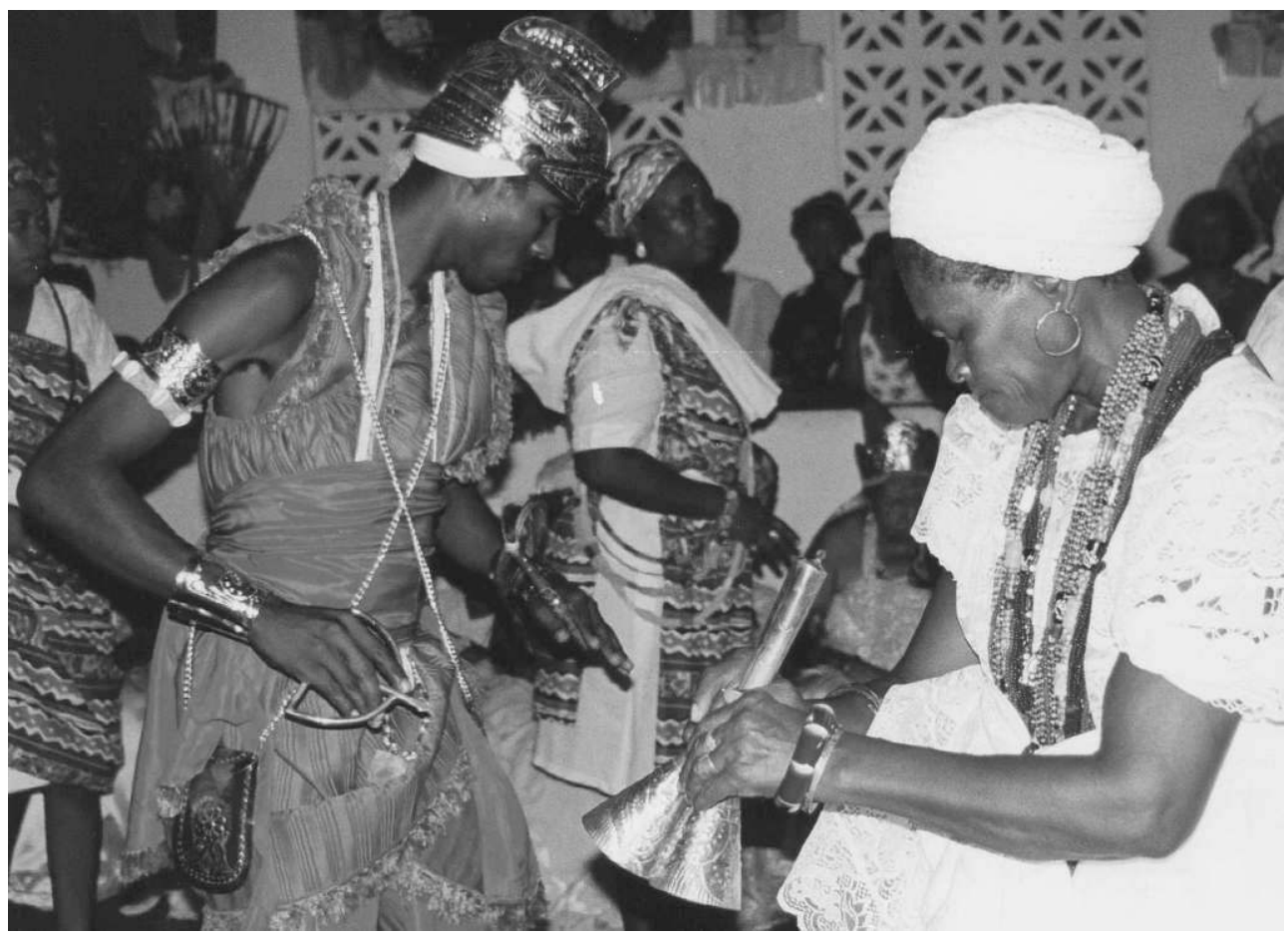

\section{Des idiophones ${ }^{4}$ spécifiques}

- La cloche sacrée adjá : cette cloche à battants, en principe agitée par la mère ou le père de saint, est utilisée dans les trois nations étudiées, mais pas dans les cérémonies pour les caboclos ; son pouvoir d'induction est forgé pendant l'initiation. Agitée à l'oreille d'un initié, elle a pour effet de déclencher la possession ; elle sert ensuite de guide sonore au possédé, puisque la possession par les orixás, voduns et inquices se déroule le plus souvent yeux fermés. Selon Nancy de Souza, initiée du célèbre terreiro (lieu de culte) Axé Opô Aganju, le son de l' adjá, lié à l'ancestralité, est caractéristique d'Oxalá ; c'est pour cette raison qu'il aurait le pouvoir d'« appeler » tous les autres orixás, puisque Oxalá est considéré comme le père de tous les orixás. Elle explique comment cet instrument déclenche, chez elle, la possession : Le volume sonore de l'adjá me déconcerte; c'est comme si j'étais complètement « désorientée » (sem rumo). Il provoque un mal-être plus grand que s'il était fort [le son de l'adjá est assez faible]. Ce son est lié au silence. Plus il est lent, plus il est faible, et pire c'est ! (entretien avec Nancy de Souza, 2000).

- La cloche gã : instrument essentiellement consacré aux divinités Omolu, Nanã, et Oxumarê ; lorsqu'il est joué dans la « maison » de ces troisdivinités, il a pour effet de déclencher la possession chez leurs initiés. D'origine Jêje, cet instrument était joué, selon Nancy de Souza, pour les rois du Dahomey.

- L'arô : ces deux cornes de buffle, entrechoquées, sont uniquement employées lors de la « fête » de la divinité Oxossi, où elles ont pour effet de déclencher la possession chez les initiés d'Oxossi, Ogum, Iansã et Oxum. L'arô est traditionnellement utilisé par l'afikodé, homme voué au culte d'Oxossi. Originaire de la nation Ketu, il est parfois utilisé dans certains terreiros appartenant à une autre nation. 
- Le kadakorô : ces fines cloches de fer, consacrées à Ogum, ont pour effet de déclencher la possession chez les initiés d'Ogum et Oxossi. C'est l'axogum -hommechargé des sacrifices rituels - qui, dans la « maison » d'Ogum, les utilise, au cours de rites privés.

- Le xeré : ce hochet en cuivre, consacré à Xangô, déclenche généralement la possession chez les initiés de cette divinité. adeptes sont toujours en position de "musiqués" - sauf en ce qui concerne certains "chants d'appel ", dans les fêtes de caboclos. Ces déclencheurs sont employés par des personnes qui ne seront vraisemblablement pas possédés, tout au moins à ce moment de la cérémonie.

\section{Autres déclencheurs}

\section{Projection de pop-corn (pipoca)}

Dans la nation Ketu, au cours de l'olubajé, cérémonie où l'on sert aux convives la nourriture pour Omolu - divinité des maladies contagieuses, notamment de la variole, dont il est atteint - la projection de pop-corn, que les adeptes nomment la «fleur d'Omolu ${ }^{5}$ ", a pour effet de déclencher la possession chez les initiés d'Omolu, de Nanã et d'Oxumarê, car tous trois sont, dit-on, originaires de la nation Jêje. Cet effet déclencheur est forgé pendant l'initiation, au cours de rites secrets. Dans la nation Jêje Mahi de Cachoeira, petite ville considérée comme le berceau bahianais de cette nation originaire de l'ancien royaume du Dahomey, où le culte de ces trois divinités est très important, Luiza da Rocha affirmait pourtant que cette pratique n'existait pas. Dans la nation Angola, lors des cérémonies pour la divinité Tempo, dont les costumes rituels, souvent faits de paille, ressemblent à ceux d'Omolu, la projection de pop-corn est fréquente, ainsi que son effet déclencheur, notamment chez les initiés de Tempo et d'Insumbo, l'équivalent angola d'Omolu.

\section{Aspersion de parfum}

Dans les trois nations étudiées, il est fréquent que des personnes de l'assistance aspergent de parfum ${ }^{6}$ les déesses Oxum et Yemanjá, lorsque celles-ci pénètrent dans le barracão (pièce principale où se tiennent les cérémonies), revêtues de leurs somptueux costumes rituels. Cette odeur forte aurait pour effet, selon certains initiés, de stimuler la survenue de la possession chez certaines personnes de l'assistance, filles ou fils de l'une de ces deux divinités.

\section{Pemba, soufflée au visage d'un initié}

La pemba est une poudre blanche crayeuse utilisée dans toutes les nations de candomblé et dont les usages rituels sont nombreux. Au cours des fêtes pour les caboclos, ainsi que dans certaines cérémonies Angola, elle est utilisée au début du rituel pour « ouvrir » la cérémonie et pour expurger le barracão de la présence éventuelle d'esprits indésirables. Soufflée au visage des membres de la communauté et des visiteurs, elle a parfois pour effet de déclencher la possession chez certains d'entre eux. 


\section{Vaporisation d'alcool (bière et cachaça ${ }^{7}$ notamment)}

14 Au cours des fêtes pour les caboclos, il est fréquent qu'un caboclo boive de la bière chaude au goulot et la vaporise ${ }^{8}$ au visage de certaines personnes susceptibles de « recevoir » un caboclo, ce qui alors a pour effet de déclencher très efficacement la possession.

\section{Accolade ou embrassade (abraço) d'un possédé}

Dans les trois nations étudiées, lorsque les divinités, incarnées par les initiés, viennent danser dans le barracão, elles ont pour coutume de serrer un à un dans leurs bras les membres de la communauté ainsi que les visiteurs. Pour les adeptes, «c'est une énergie qui se transmet » et qui a pour effet de déclencher la possession chez certaines personnes. Cette pratique se retrouve aussi au cours des cérémonies pour les caboclos.

\section{Vue d'un possédé exécutant une gestuelle particulière}

La possession par les divinités africaines s'inscrit dans un carcan mythologique très étroit. Une fois possédés par leurs divinités respectives, les initiés doivent accomplir un ensemble de danses comportant des gestuelles très spécifiques. Certains fils d'Oxumarê, par exemple - rappelons que cette divinité est assimilée au serpent, le Dan des Fon du Dahomey - se baissent jusqu'à terre, ingurgitent de l'eau, préalablement déposée dans une bassine, se relèvent en mimant les contorsions du serpent et vaporisent cette eau au milieu du barracão. La vision de cette scène relativement rare suffit à déclencher la possession chez certains initiés. De même, le «bain d'Oxum »- moment où Oxum, déesse de la beauté, mime de prendre son bain en s'admirant dans son miroir puis, telle une Vénus anadyomène, sort de l'eau en se relevant de façon très sensuelle - a pour effet de déclencher la possession chez certains initiés, notamment ceux d'Ogum, Oxossi et Xangô, divinités masculines qui, selon les légendes, ont succombé à ses irrésistibles charmes. Nancy de Souza parle à ce propos d'une "transe émotionnelle» (transe emocional) qui serait suscitée par un geste particulier ou un chant spécifique. Selon elle, son déclenchement dépend aussi du degré d'attention de la personne ; il faudrait un « cumul d'énergie » suffisant pour déclencher ce type de transe. Elle évoque aussi une "transe esthétique » (transe estético), concluant que l'émotion suscitée comporte une dimension esthétique ; c'est la beauté de la scène - ou du chant - qui, suscitant une émotion très forte, déclencherait alors la possession. Notons que cette informatrice est la seule adepte $\mathrm{du}$ candomblé avec laquelle nous avons pu aborder le sujet de la possession de manière aussi " fructueuse " ${ }^{9}$. En effet, quand il s'agit d'aborder le vécu de la possession, la règle de l'amnésie - ou plus précisément le « devoir » d'amnésie - qui fait suite à la possession, rend presque toujours l'investigation difficile, voire impossible.

\section{Ingestion de jurema}

17 La jurema est une plante dont on utilise les feuilles pour préparer un breuvage du même nom, tenu pour faiblement hallucinogène; très apprécié des caboclos, sa composition faite de sang et de vin notamment - varie d'un lieu de culte à l'autre. Au cours des fêtes pour les caboclos, lorsque ces derniers se sont déjà "manifestés », on les emmène dans leur « cabane " - véritable cabane préparée à l'occasion de la fête, qui est généralement décorée d'une multitude de fruits et de divers symboles qui leur sont associés - devant 
laquelle ils vont boire la jurema et l'offrir aux personnes présentes. Dans certains cas, l'ingestion est suivie de la possession et de la venue d'un nouveau caboclo. Notons toutefois que l'ingestion et la possession sont simultanées, ce qui infirme l'hypothèse selon laquelle la substance tenue pour hallucinogène serait à l'origine de la possession. S'il y a induction, elle est de nature symbolique et non physiologique.

\section{$L^{\prime}$ « entrée en transe »}

L'« entrée en transe » est décrite par un grand nombre d'expressions vernaculaires, parmi lesquelles :

- Bolar no santo : cette expression s'applique en principe à des personnes qui sont possédées pour la première fois - la « crise de pré-possession » décrite par Roger Bastide - ou à certains prétendants à l'initiation qui n'ont pas encore décidé de se soumettre au processus initiatique. La personne est soudain « prise » par la divinité - ou parfois, dit-on, par l'un de ses exus - et tombe à terre violemment. Elle est alors conduite dans le roncó - pièce secrète où se déroule la phase de réclusion initiatique - et l'officiant " parle en langue » (troca língua ) avec l'entité concernée pour savoir quelle est sa volonté et éventuellement la raison pour laquelle elle vient d'infliger cette " punition » (castigo) à la « matière » (le possédé) ; en effet, certains initiés, qui n'ont pas accompli leurs « obligations » rituelles envers leur divinité, sont parfois sujets à une « crise » de ce type. Certains terreiros organisent parfois un toque de bolar, c'est-à-dire une cérémonie spécialement destinée à déclencher ce type de possession parmi les visiteurs, ce qui assure à la communauté le recrutement, à plus ou moins court terme, de nouveaux novices.

- L'« entrée en transe » la plus courante est décrite, dans les trois nations étudiées, par les expressions suivantes : «tomber dans le saint » (cair no santo), « tourner de saint » (virar de santo), « le saint est descendu »(o santo baixou), « le saint (l')a pris »(o santo pegou). On peut remarquer certains stéréotypes comportementaux. Lorsqu'il s'agit d'une personne de l'assistance, l'entrée en transe est souvent précédée de signes annonciateurs : l'individu semble se déconnecter du contexte cérémoniel, son regard devient vague, il baille, se frotte les mains. Certains se lèvent pour sortir du barracão, afin d'échapper à une possession pourtant inéluctable. C'est souvent à ce moment que l'individu est soudain " pris » par sa divinité. Dans tous les cas, qu'il s'agisse de visiteurs ou d'initiés participant activement à la cérémonie, cette phase s'accompagne de tremblements des épaules et de la tête. Le corps est courbé et les mains se joignent derrière le dos ou sur le flanc. L'individu est alors pris en charge par une ekede, femme dont le rôle est de s'occuper des possédés. Mis à part le contexte liturgique - chants ou formules rythmiques spécifiques - le premier signe qui permet d'identifier la divinité en question est le cri poussé par le possédé : on dit alors que le saint «a répondu » (o santo respondeu). Chaque divinité possède en effet un cri caractéristique, que l'on nomme ilá ${ }^{10}$.

- Tomar barravento : cette expression, vraisemblablement originaire de la nation Angola - le terme barravento désigne en effet une formule rythmique caractéristique de cette nation s'applique le plus souvent, mais pas exclusivement, à la possession par les caboclos. On dit que la personne « prend barravento » (toma barravento) lorsque l'entité s'approche d'elle pour s'en emparer ; elle perd l'équilibre, titube, tourne sur elle-même, se passe à plusieurs reprises la main sur le visage, ses yeux sont parfois exorbités et son expression semble manifester un effroi très particulier. Elle cherche à se tenir à tout ce qui l'entoure - des personnes de l'assistance, un mur - pour éviter la chute. Elle tente de sortir du barracão pour 
échapper à la possession, car ce comportement est censé exprimer sa lutte contre la venue de l'entité, qu'il s'agisse d'un caboclo ou d'une divinité. Au bout de quelques instants, voire de quelques minutes, soit la personne revient progressivement à elle, soit, ce qui est plus fréquent, elle « succombe » à la possession. À ce propos, ajoutons que certains initiés, par exemple en visite dans un autre terreiro à l'occasion d'une cérémonie rituelle, emploient certains « subterfuges »- comme porter une gousse d'ail sur soi - pour tenter d'éviter la survenue de la possession.

Fig. 2 : « Entrée en transe ». Camaçari (Bahia), 2003. Photo Xavier Vatin.

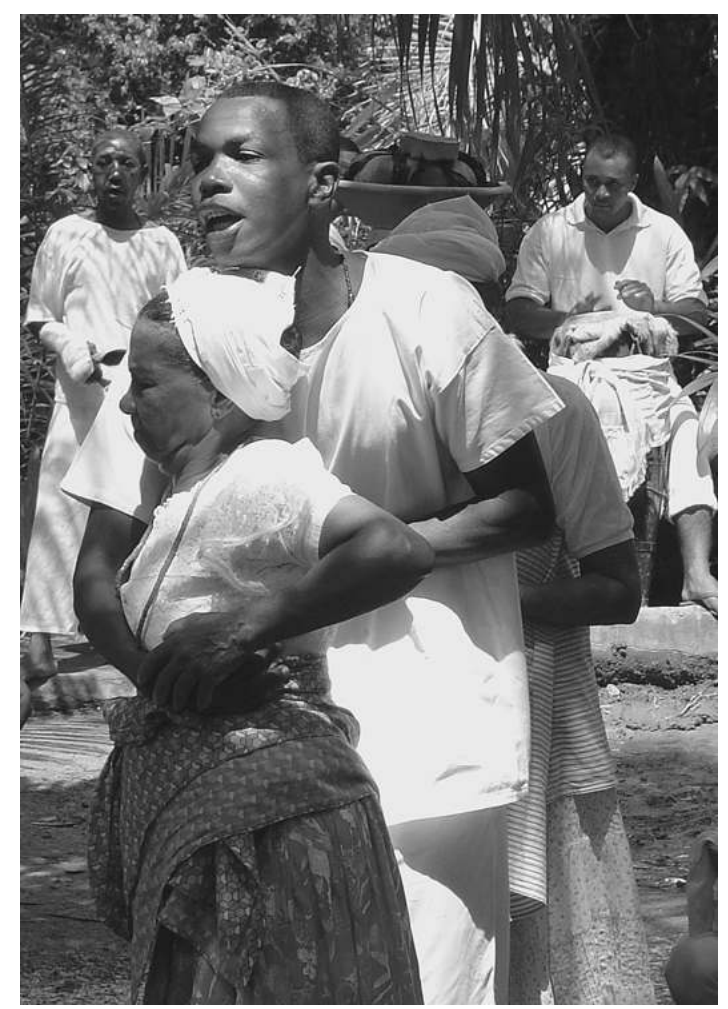

\section{Le comportement du possédé}

La possession - décrite par l'expression courante estar de santo, "être de saint ", quand il s'agit des orixás Ketu, des voduns Jêje ou des inquices Angola - prend des formes très diverses selon le type d'entité censé s'être incarné.

On peut dire d'emblée que le comportement des divinités africaines se distingue nettement de celui des caboclos ou des exus. La possession par les orixás, voduns ou inquices est essentiellement une possession dansée, une "transe du corps", pour reprendre l'expression de Roberto Motta (1990). Chaque divinité exprime et mime par la danse les légendes qui lui sont associées. Les divinités africaines ne sont pas pour autant muettes; toutes s'expriment vocalement au moins par le cri. Dans ce type de possession, les possédés sont tous "musiqués", dans le sens où les tambours accompagnent toujours leurs danses. Ils ont en général les yeux fermés et ne parlent qu'en de rares occasions.

21 Ces caractéristiques générales ne doivent toutefois pas occulter les différences significatives qui distinguent orixás, voduns et inquices. Même si ces trois types de possession peuvent s'apparenter à la catégorie "transe de possession ", utilisée par 
Gilbert Rouget, on constate que certaines divinités manifestent une attitude musicale active, qui remet en question la typologie structuraliste selon laquelle, au cours d'une «transe de possession », le possédé est exclusivement «musiqué » et en en aucun cas «musiquant» de sa propre transe. En effet, tous les voduns de la nation Jêje chantent au cours des cérémonies rituelles : le possédé se présente face aux tambours et entonne le chant de son choix, qui est en général repris par le chef de culte, puis par les membres de la communauté ; il se retrouve quelques secondes plus tard en position de "musiqué » puisque les tambours et le chant, qu'il a lui-même préalablement entonné, vont accompagner sa danse. En principe, les orixás et les inquices, eux, ne chantent pas. Pendant les cérémonies, ils ne s'expriment donc vocalement que par le cri qui caractérise chacun d'entre eux.

La possession par les caboclos présente des caractéristiques bien différentes. Même si la danse est, pendant les cérémonies, un moyen d'expression privilégié du caboclo, ce dernier possède d'autres manières spécifiques de s'exprimer et d'interagir avec les membres de la communauté et de l'assistance. La possession par un caboclo est à la fois une «transe du corps » et une «transe de la parole » : en effet, celui-ci ne se contente pas d'entonner à voix basse les chants de son choix, tel un vodum de la nation Jêje, il fait souvent office de véritable chanteur soliste, même lorsqu'il danse simultanément. Il converse avec les autres caboclos et avec l'assistance, dans un portugais qui lui est propre et qu'on qualifie de embolado (littéralement "emmêlé »). Il a pour coutume de fumer le cigare et de boire de la jurema ainsi que de la bière chaude au goulot, qu'il offre aussi à l'assistance. À la fin des cérémonies, le caboclo invite un à un, d'un appel du pied ou de l'épaule, les membres de l'assistance à danser avec lui, ce que l'on nomme alors samba de caboclo. L'interaction entre les caboclos et l'assistance est donc bien plus importante que dans le cas des divinités africaines.

Les exus, qui chantent rarement, dansent essentiellement sur un rythme de samba et, à l'instar des caboclos, invitent les participants de la fête à danser avec eux. Ils ont pour coutume de boire des alcools forts (cognac, whisky, cachaça) et, lorsque ces spiritueux viennent à manquer, de la bière chaude. Ils fument également le cigare ou la cigarette. Leur comportement est en général provocateur ; ils n'hésitent pas à faire des propositions très indécentes à certains visiteurs.

Les cérémonies pour les caboclos, comme celles pour les exus, possèdent un caractère particulièrement festif, voire licencieux, où l'improvisation tient une place bien plus importante que dans les cérémonies pour les orixás, inquices ou voduns, ce qui explique sans doute en partie la faveur dont elles jouissent parmi de nombreux Bahianais. En outre, ajoutons que, lors de ces fêtes, les membres de l'assistance ont presque toujours l'occasion de consulter individuellement un caboclo ou un exu.

\begin{tabular}{|l|l|l|l|l|l|}
\hline Comportement & Orixás & Voduns & Inquices & Caboclos & Exus \\
\hline Danse & oui & oui & oui & oui & parfois \\
\hline Chant & non & oui & rarement & oui & rarement \\
\hline Cri caractéristique & oui & oui & oui & oui * & rires \\
\hline Usage de la parole & rare & rare & rare & oui & oui \\
\hline
\end{tabular}




\begin{tabular}{|l|l|l|l|l|l|}
\hline Jurema & non & non & non & oui & non \\
\hline Bière & non & non & non & oui & oui \\
\hline Cachaça & non & non & non & non & oui \\
\hline Cigare / cigarette & non & non & non & oui & oui \\
\hline
\end{tabular}

* Les cris des caboclos ne sont pas aussi caractéristiques que ceux des divinités africaines ; il est donc difficile d'identifier à coup sûr tel ou tel caboclo par son cri.

Le tableau ci-dessus permet de comparer certaines caractéristiques du comportement du possédé en fonction de l'entité qu'il est censé incarner.

\section{Le retour à l'« état normal »}

\section{Retour « déclenché »}

Dans certaines circonstances - quand il s'agit d'un initié provenant d'un autre terreiro ou pour mettre un terme à une possession qui semble ne pas vouloir se terminer - le retour à l'« état normal » est déclenché par le chef de culte ou par son substitut; on utilise à cet égard l'expression " expédier le saint » (despachar o santo). La personne chargée de cette tâche délicate, après avoir emmené le possédé dans le roncó, emploie des techniques corporelles ainsi que des formules verbales spécifiques, dont la teneur est secrète.

\section{Retour « indirect »}

Le retour à l'« état normal » est souvent précédé par la venue de l'erê, forme infantile de la divinité principale. Les erês, qui ne cessent de faire toutes sortes de pitreries, peuvent rester plusieurs heures après la fin de la cérémonie, et reviennent souvent le matin suivant. Il faut souvent les « expédier " (despachar) avec autorité pour se débarrasser de leur présence encombrante. La plupart d'entre eux ne veulent pas " partir » et se mettent alors à pleurer ou tentent de s'échapper. La venue de l'erê se raréfie généralement avec l'ancienneté de l'initié.

\section{Retour « direct »}

Dans d'autres cas, le retour à l' "état normal " ne nécessite pas de phase transitoire, comme celle représentée par la venue de l'erê. Le retour « direct » est l'apanage des vieux initiés. En ce qui concerne la possession par les caboclos et les exus, le retour est toujours direct et n'est pas accompagné de phase transitoire.

29 La plupart des adeptes, après la possession, se plaignent de faim et soif intenses ; certains sont atteints de vertiges; tous déclarent n'avoir aucun souvenir de ce qui s'est passé pendant la possession. 


\section{Les possessions au quotidien}

La possession ne survient pas exclusivement dans un contexte cérémoniel. Elle peut se manifester dans le cadre domestique, surtout en ce qui concerne les caboclos et les exus qui, du fait de leur aptitude à la parole, sont fréquemment sollicités lors de consultations plus ou moins formelles.

Prenons le cas de Jacira, fille d'Obaluaiê et Iansã, initiée dans un terreiro de nation Ketu. Ayant pris des distances avec sa communauté d'origine, elle « reçoit » régulièrement, à son domicile, une exua (féminin de exu) du nom de Maria Formosa, que de nombreuses personnes viennent consulter pour des problèmes très divers. Formosa possède, dans l'arrière-cour de la maison de Jacira, une petite "maison » qui lui est exclusivement consacrée, dans laquelle les consultations ont lieu. Lorsque plusieurs "clients " sont déjà arrivés, Jacira les invite à se regrouper autour d'elle, à l'entrée de la maison de Formosa. Assise à l'intérieur, Jacira se concentre et prononce quelques paroles rituelles; après quelques minutes, Formosa s'empare d'elle soudainement en poussant de vigoureux éclats de rires caractéristiques qui annoncent sa venue. Les yeux exorbités, elle vient saluer une à une les personnes présentes ; la consultation peut alors commencer.

Ce qui nous intéresse tout particulièrement dans cet exemple, c'est l'absence de tout " déclencheur » semblable à ceux que nous avons évoqués plus haut : aucun chant, aucun rythme, aucun son de cloche, aucune ingestion - même symbolique - d'une quelconque substance ne vient déclencher la possession. C'est par la concentration, dit-elle, et les invocations qu'elle-même profère, que Jacira suscite la venue de Formosa. Cette possession ne nécessite donc aucun déclencheur externe. 
Fig. 3. Danse du caboclo Boiadeiro. Itapitanga (Bahia), 2004.

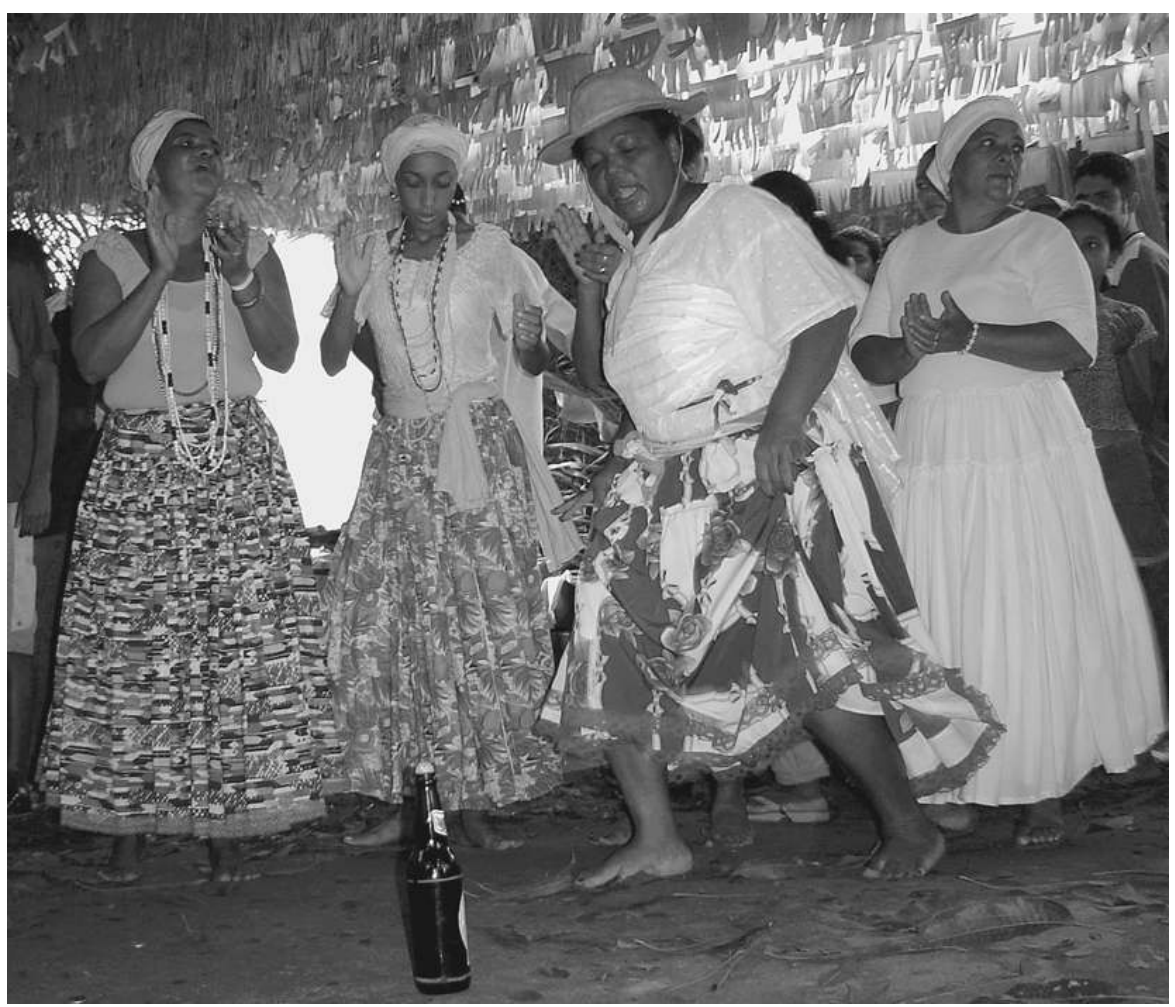

Photo Xavier Vatin.

cette pratique du chamanisme, du moins si on le conçoit dans une perspective structuraliste. Cet exemple contribue à montrer, parmi de nombreux autres, que des pratiques longtemps tenues pour diamétralement opposées - possession et chamanisme présentent probablement autant de caractéristiques communes que de divergences profondes. formes, de façon extrêmement courante à Bahia. Échappant à toute tentative de typologie globalisante, ces possessions, singulières et plurielles, s'inscrivent dans un «empire de l'imaginaire " marqué par les interpénétrations de civilisations, le métissage et le pluralisme des pratiques rituelles et comportementales. L'impasse faite sur ces pratiques - que certains traditionalistes, initiés mais aussi ethnologues, qualifient volontiers d'hérétiques - nous semble plus révélatrice des a priori méthodologiques et idéologiques de certains chercheurs, que de leur plus ou moins grande représentativité et légitimité dans un continuum religieux afro-brésilien extrêmement plural. En effet, soucieux de valider des théories parfois trop générales, ceux-ci ont privilégié une démarche hypothético-déductive conduisant à un formalisme typologique, mais également à la validation d'un modèle d'orthodoxie, qu'une analyse minutieuse de la diversité des pratiques rituelles et musicales remet en question.

Expression symbolique d'un système fait de "branchements " multiples (cf. Amselle 2001), les candomblés de Bahia oscillent entre un «métissage culturel » ouvertement assumé et la recréation militante d'une Afrique mythique, à la fois proche et lointaine. Dans cet univers religieux, les relations de la musique et de la possession oscillent 
également entre un pluralisme rituel et comportemental particulièrement complexe, difficile à saisir, et le désir de conformité envers des modèles d'orthodoxie et d'orthopraxie - issus de l'alliance entre un segment du culte extrêmement minoritaire et certains ethnologues -, gages d'une «africanité idéale » qui est, aujourd'hui plus que jamais, un enjeu de reconnaissance sociale et institutionnelle.

\section{BIBLIOGRAPHIE}

AMSELLE Jean-Loup, 1999, Logiques métisses. Anthropologie de l'identité en Afrique et ailleurs. Paris : Bibliothèque Scientifique Payot (1 $1^{\mathrm{e}}$ éd. 1990).

AMSELLE Jean-Loup, 2001; Branchements. Anthropologie de l'universalité des cultures. Paris :

Flammarion.

ARGYRIADIS Kali , 1999, «Une religión vivante. Continuité et complémentarité des pratiques cultuelles havanaises ». L'Homme $151: 21-46$.

AUGÉ Marc, 1988, Le dieu objet. Paris : Flammarion.

BASTIDE Roger, 1958, Le Candomblé de Bahia (rite Nagô). Paris : Mouton.

BASTIDE Roger, 1972, Le rêve, la transe et la folie. Paris : Flammarion.

BASTIDE Roger, 1995, Les religions africaines au Brésil. Contribution à une sociologie des interpénétrations de civilisations. Paris : Presses Universitaires de France (1e éd. 1960).

BASTIDE Roger, 1997, Le sacré sauvage. Paris : Stock (1 éd. 1975).

BOYER-ARAUJO Véronique, 1993, « 'Les traditions risquent-elles d'être contaminées ?' Paradigmes scientifiques et orthodoxie religieuse dans les cultes de possession au Brésil ». Journal de la Société des Américanistes LXXIX : 67-90.

CAPONE Stefania

1999 La quête de l'Afrique dans le candomblé. Pouvoir et tradition au Brésil. Paris : Karthala.

CARNEIRO Edison, 1937, Negros Bantus. Notas de ethnographia religiosa e de folk-lore. Rio de Janeiro : Civilização Brasileira.

CARNEIRO Edison, 1961, Candomblés da Bahia. Rio de Janeiro : Tecnoprint (1éd. 1948).

COSSARD Gisèle, 1970, Contribution à l'étude des candomblés au Brésil. Le candomblé angola.Th., Paris : Faculté des Lettres et Sciences Humaines.

FRIGERIO Alejandro, 1989, « Levels of possession awareness in Afro-Brazilian religions ». Association for the Anthropological Study of Consciousness Quarterly 5 : 5-11.

FRY Peter, 1984, «Gallus africanus est, ou, como Roger Bastide se tornou africano no Brasil ». Folha de São Paulo. São Paulo : Folhetim, 15 juillet 1984 : 11-14.

HELL Bertrand, 1999, Possession et chamanisme. Les maîtres du désordre. Paris : Flammarion.

LEVI-STRAUSS Claude, 1958, «L'efficacité symbolique », in Anthropologie Structurale. Paris : Plon :

213-234. 
LEVI-STRAUSS Claude, 1995, La pensée sauvage. Paris : Pocket (1éd. 1962).

MARY André, 1994, « Bricolage afro-brésilien et bris-collage post-moderne », in Roger Bastide ou le réjouissement de l'abîme, sous la direction de Philippe Laburthe-Tolra, Echos du colloque tenu à Cerisay-la-Salle du 7 au 14 septembre 1992. Paris : L'Harmattan : 85-98.

MERRIAM Alan P., 1951, Songs of the afro-bahian cults. An ethnomusicological analysis. Th., Evanston : Northwestern University.

MERRIAM Alan P., 1964, The Anthropology of Music. Evanston : Northwestern University.

MOTTA Roberto, 1990, « Transe du corps et transe de la parole dans les religions syncrétiques du Nordeste du Brésil ». Cahiers de l'Imaginaire 5 et $6: 47-62$.

PIERSON Donald, 1971, Brancos e prêtos na Bahia (estudo de contacto racial). São Paulo, Editora Nacional (original : Negroes in Brazil : A study of race contact at Bahia. Chicago : University of Chicago Press, 1942).

PINTO Tiago de Oliveira, 1992, «La musique dans le rite et la musique comme rite dans le candomblé brésilien ». Cahiers de musiques traditionnelles 5. Genève : Ateliers d'ethnomusicologie : 53-70.

PRINCE Raymond (éd.), 1968, Trance and possession states. Montreal : R.M.Bucke Memorial Society, 4-6 March 1966.

RAMOS Arthur, 1940, 0 negro brasileiro ( $1^{\circ}$ vol. : Ethnografia religiosa). São Paulo, Rio, Recife, Porto Alegre : Editora Nacional (1 ${ }^{\mathrm{e}}$ éd. 1934).

ROCHA Luiza Franquelina da, 1997, « Nação Jeje », in Segundo encontro de nações de candomblé. Salvador : CEAO, Universidade Federal da Bahia : 68-83.

RODRIGUES Nina, 1977, Os africanos no Brasil. São Paulo : Editora Nacional (1 ${ }^{\mathrm{e}}$ éd. 1906).

ROUGET Gilbert, 1990, La musique et la transe. Esquisse d'une théorie générale des relations de la musique et de la possession. Paris : Gallimard (1éd. 1980).

SERRA Ordep, 1995, Águas do Rei. Petrópolis/Rio de Janeiro : Vozes/Koinonia.

TURNER Victor W., 1990, Le phénomène rituel. Structure et contre-structure. Paris : Presses Universitaires de France (1 éd. 1969).

VATIN Xavier, 1999, Candomblé de Angola. Musique Rituelle Afro-Brésilienne. Paris : CD Inédit-Maison des Cultures du Monde.

VATIN Xavier, 2001, Étude comparative de différentes nations de candomblé à Bahia, Brésil. Thèse de Doctorat en Anthropologie Sociale et Ethnologie, Paris : EHESS.

VATIN Xavier, 2005a, Rites et musiques de possession à Bahia. Paris : L'Harmattan

VATIN Xavier, 2005b, « Dandalunda, maimbanda coquê. Les musiques du candomblé : du rite africain au carnaval bahianais ", in Brésil, l'héritage africain. Paris : Dapper : 152-169.

VERGER Pierre Fatumbi, 1982, Orisha. Les Dieux Yorouba en Afrique et au Nouveau Monde. Paris : Métailié (éd. originale brésilienne 1981).

VIANA FILHO Luis, 1988, O negro na Bahia. Rio de Janeiro : Nova Fronteira (1 ${ }^{\mathrm{e}}$ éd. 1946).

WAFER Jim, 1991, The Taste of Blood. Spirit Possession in Brazilian Candomblé. Philadelphia : University of Pennsylvania Press. 


\section{ANNEXES}

\section{Glossaire}

$(y=$ yoruba $; \mathrm{p}=$ portugais $; \mathrm{f}=$ fon $; \mathrm{b}=$ bantou $)$

Adjá (y)

Cloche double ou triple à battants internes, généralement agitée par le chef de culte à l'oreille des initiés pour déclencher la possession. Elle sert ensuite de guide sonore et spatial au possédé.

Agogô (y)

Instrument composé de deux cloches métalliques de taille différente, percutées à l'aide d'une baguette métallique. Il exécute le rythme qui sert de base aux tambours et aux chants.

Caboclo (p)

Métis de l'indien et du blanc;

Esprit d'indien, considéré dans le candomblé comme le dono da terra, le « maître des terres ». Il se manifeste par la possession dans des cérémonies qui lui sont propres.

Cantiga

Chant investi d'un pouvoir particulier et ayant fréquemment pour de fundamento (p) effet de déclencher la possession.

Erê (y)

Forme infantile de l'orixá. L'état d'erê, déclenché au cours de l'initiation, est une étape transitoire fréquente entre la possession par l'orixá et le retour à l'état de conscience ordinaire. Un erê parle et se comporte comme un jeune enfant.

Exu (y)

Dans la tradition yoruba, Exu est un esprit malicieux qui sert d'intermédiaire entre les hommes et les orixás. A ce titre, il fait l'objet d'attentions très particulières, notamment avant les cérémonies (padê de Exu). Le terme exu désigne par ailleurs les esprits qui sont les « esclaves » (escravos) des orixás et « travaillent » pour eux. Lorsqu'ils se manifestent par la possession, dans un contexte cérémoniel ou domestique, les gens viennent alors les consulter.

Gã (f)

Cloche métallique simple à battant externe dont la fonction est similaire à celle de l'agogô. Inquice (b)

Transcription brésilienne du terme bantu nkisi, qui désigne les divinités du panthéon Angola.

Jurema

Plante dont on utilise les feuilles pour préparer un breuvage du même nom, tenu pour faiblement hallucinogène ; très apprécié des caboclos, sa composition (sang, cachaça ou vin notamment) varie d'un lieu de culte à l'autre. Jurema est aussi le nom d'une cabocla. 
Orixá (y)

Divinité, ancêtre divinisé (santo). Ce mot yoruba, originaire de la nation Ketu, est utilisé couramment par les membres de toutes les nations de candomblé (même si chacune d'elles conserve un terme spécifique pour désigner un concept similaire).

Padilha

Esprit d'exu féminin, qui se manifeste dans certains candomblés ainsi que dans l'umbanda (où l'on trouve plus souvent son équivalent Pomba Gira); elle prend différents noms propres (Maria Padilha, Maria Formosa) et revêt toujours la forme d'une prostituée à l'allure provocante.

Toque (p)

Terme générique signifiant formule rythmique.

Toque

Formule rythmique investie d'une force particulière et associée, à

de fundamento $(\mathrm{p})$

l'exception de l'adarrum, à une divinité spécifique. (ex. : alujá de Xangô).

Vodum (f)

Nom donné aux divinités dans la nation Jêje.

Vunji (b)

Forme infantile de l'inquice, dans la nation Angola ; équivalent de erê.

\section{NOTES}

1. Mentionnons à ce propos l'ouvrage de référence de Gilbert Rouget: La musique et la transe. Esquisse d'une théorie générale des relations de la musique et de la possession (1990).

2. Traduction de nação, terme censé déterminer les origines ethniques et culturelles prédominantes d'un lieu de culte. On distingue différentes "nations» de candomblé, parmi lesquelles subsistent à Bahia : les nations Ketu et Ijexá, d'origine linguistique yoruba, également connues sous le terme générique de Nagô ; la nation Jêje, d'origine linguistique fon ; les nations Angola et Congo, d'origine linguistique bantu (kimbundu et kikongo). Chaque nation de candomblé se distingue principalement par un panthéon spécifique - composé d'orixás, voduns ou inquices - et par une « langue » qui atteste de son lien avec les cultures d'origine. Cette " langue » se retrouve à des degrés divers dans les chants, les prières et la terminologie rituelle.

3. On peut entendre un chant de ce type, enregistré au cours d'une cérémonie rituelle, dans le disque Candomblé de Angola. Musique Rituelle Afro-Brésilienne (Inédit, Maison des Cultures du Monde, 1999 : plage 7, « Entrées en transe »).

4. Dans le candomblé, comme dans de nombreux cultes de possession, la plupart des idiophones utilisés sont avant tout des « instruments rituels».

5. Flor de Omolu; on dit qu'elle calme et rafraîchit le corps brûlant et couvert de plaies de cette divinité.

6. Le parfum est souvent mélangé avec des fleurs et du riz; ce mélange est alors distribué à l'assistance avant l'arrivée des divinités.

7. Cachaça est le nom donné, au Brésil, à l'alcool de canne à sucre.

8. L'alcool n'est pas simplement « craché », mais plus exactement « vaporisé » - à Bahia, le verbe utilisé est barrufar - pratique que l'on retrouve dans de nombreux rituels de possession, notamment à Cuba. 
9. Au sujet de Nancy de Souza, il est important de noter sa "double appartenance ", au candomblé Ketu d'une part, à la Fondation Pierre Verger de l'autre, lieu où elle a accès, depuis de nombreuses années, à une immense littérature sur des sujets qui la passionnent, tels que les cultes de possession en Afrique, au Brésil, à Cuba et en Haïti. Nancy de Souza est une inestimable collaboratrice, dont il faut toutefois constamment analyser le discours à l'aune de cette double appartenance et de ce double savoir, traditionnel et académique.

10. Le cri, dans son contexte cérémoniel, est, selon Gilbert Rouget, « une manifestation très caractéristique de la transe » (Rouget, 1990 : 217). Pour une analyse détaillée de la nature et des fonctions du cri dans le candomblé, voir Vatin (2005a : 113-121).

\section{RÉSUMÉS}

Les candomblés de Bahia ont fait l'objet de nombreuses études dans divers domaines des sciences sociales. Pourtant, l'ethnographie traditionnelle propose encore fréquemment une vision du candomblé statique et monolithique, bien éloignée de la réalité quotidienne de pratiques rituelles en constante métamorphose. En privilégiant une démarche inductive, cet article tente de montrer comment s'élaborent, dans les candomblés de Bahia, les relations très diverses de la musique et de la possession, selon le contexte rituel et le type d'entité censé s'incarner. Le pluralisme rituel et comportemental ainsi observé remet partiellement en cause certaines typologies parfois trop formelles, fondées sur des oppositions binaires - musiquant/musiqué, chamanisme/possession. En outre, loin des clichés ethnocentriques et évolutionnistes, notamment remis au goût du jour par le mouvement new age, ce pluralisme vient confirmer que le lien entre musique et possession est de nature symbolique et non physiologique. En ce sens, leurs relations sont plus complexes que certains ont coutume de croire.

\section{INDEX}

Index géographique : Bahia, Brésil

\section{AUTEUR}

\section{XAVIER VATIN}

Xavier Vatin est ethnomusicologue, docteur en anthropologie sociale et ethnologie à l'EHESS. Il a passé plus de dix ans sur le terrain, à Bahia. Membre associé du Laboratoire Langues-MusiquesSociétés (UMR 8099 du CNRS), il dirige actuellement, à l'Université Fédérale de Bahia, le projet Nzila, consacré aux traditions religieuses et musicales d'origine bantu. Sa démarche consiste à tenter de faire converger anthropologie et ethnomusicologie afin d'étudier certains phénomènes de métissage, religieux, linguistique et musical. 